\title{
Performance Comparison of Guided-Wave Architectures for Space-Division Photonic Switching
}

\author{
Luigi Savastano, Guido Maier, Mario Martinelli \\ CoreCom \\ Via Colombo 81 \\ 20133 Milan, Italy \\ \{t_luisav,maier,martinelli\}@corecom.it
}

\author{
Achille Pattavina \\ Politecnico di Milano, \\ P.za Leonardo da Vinci, 32 \\ 20133 Milan, Italy \\ pattavina@elet.polimi.it
}

\begin{abstract}
The paper ${ }^{1}$ presents and compares various unicast nonblocking architectures to be used into space-domain photonic switching networks. All the analyzed architectures have been evaluated and compared considering a possible physical implementation based on guided-wave structures realized with integrated optics technology. Some properties including number of switching elements required, blocking performance, number of waveguide crossovers, system attenuation, and signal-to-noise ratio are evaluated and analyzed. The main purpose of this work is to review the stateof-the-art of optical guided-wave space-switching architectures and to provide a relevant set of technical elements useful in the selection of architectures to be used in all-optical cross-connect implementation.
\end{abstract}

\section{Introduction}

Recently, the growth of network traffic has stimulated the deployment of long-haul optical network systems which employ wavelength-division multiplexing (WDM) to achieve enormous transport capacity. Such systems, having tens of wavelengths per fiber with each wavelength modulated at $2.5 \mathrm{~Gb} / \mathrm{s}, 10 \mathrm{~Gb} / \mathrm{s}$ or more [25], rely mainly upon electronics to implement the switching functions. In every switching node, optical signals are converted to electrical form (O/E conversion), switched electronically and converted back to optical form (E/O conversion). Switching systems that execute these operations are called OEO crossconnects. Although electronic switching is highly reliable, it has many disadvantages as the dependence of switching hardware upon data bit-rate and transmission protocol, and NIS high costs due to $\mathrm{E} / \mathrm{O}$ and $\mathrm{O} / \mathrm{E}$ conversion devices. The transition of the switching functions from electronics to optics with the deployment of all-optical (OOO) cross-connects will potentially reduce the network-equipment complexity and increase the flexibility, provided that the cost of OOO switches will be competitive with the cost of their OEO counterparts. The main cost advantages of the OOO solution can be envisioned in the absence of $\mathrm{E} / \mathrm{O}(\mathrm{O} / \mathrm{E})$ converters and in their transparency to the signal format. As a matter of facts, optoelectronic conversion represents an important cost component in today networks [19]. Moreover, several subsystems and components of OEO switches are subject to be substituted at any protocol or bit-rate variation.

The core of an OOO cross-connect is an optical switch that is independent of data rate and protocol. Various technologies, e.g. microelectromechanical systems (MEMS) [15], electrooptical [5], thermooptical [3], liquid-crystal [6], bubble-jet [28] and acoustooptical [21], have been proposed and studied for realization of optical switches. All these technologies can be subdivided into two large categories: free-space and guided-wave systems. For example, MEMS systems belong to the free-space category while electrooptical and thermooptical switches are guided-wave systems.

This paper has two main purposes: first, the identification of some evaluation criteria based on specific performance parameters to compare different guided-wave switching architectures; second, the presentation of an overview of the main guided-wave architectures currently state-of-the-art in technical literature. The first aspect is analyzed in section 2, while architectures are described in section 3. In this last section, after a brief description of the technology, the selection of switching architectures is characterized by providing for each case formulas to evaluate various parameters, focusing the aspects that are more peculiar for the given technology. Up to now, several guided- 
wave architectures have been proposed for optical space switching. We have collected them in this unified framework and we have calculated the parameters we have considered significative to allow a technical comparison among different possible architectural options for OOO implementation.

Although some described architectures have multicasting or broadcasting capability, in this paper we have focused our attention only on space-domain switching networks with point-to-point connection capacity.

\section{Performance parameters}

Optical switching architectures can be compared using as benchmark four different classes of characteristics: blocking properties, physical structure, signal-transfer impairments and cost. Let us introduce for each class the parameters we will use in Sec. 3, briefly discussing them oneby-one in this section to form a qualitative point of view.

\subsection{Blocking properties}

According to their switching capability, switching networks can be subdivided into two categories: blocking and non-blocking [20]. A network is said to be non-blocking if an unused input port can always be connected to any unused output port. Thus, a non-blocking network is capable to realize every permutation of input ports on output ports. If at least one of these permutations can never be realized, the network is said to be blocking. Most applications require non-blocking architectures.

Non-blocking networks can be further distinguished in three subclasses according to their dynamic behavior in the transitions from a switching state to another. In Rearrangeable Non-Blocking (RNB) architectures the setup of a new connection between an unused input and an unused output may require the reconfiguration of the entire switching network, with rearrangement of other already active connections. Since existing connections must be interrupted, though only for the switching time, rearrangeability is often considered unacceptable by operators. Strict-sense NonBlocking (SNB) and Wide-sense Non-Blocking (WNB) architectures can always setup a new connection between an unused i/o pair, regardless of the current switching state, thus avoiding rearrangements. WNB can achieve this feature only provided that any new connection is routed according to a specific setup rule. In SNB switching networks, any free route can be assigned to a new connection, indifferently. There is generally a trade-off between switching capability and complexity: the avoidance of connectionrearrangement disruptions is paid in terms of a higher number of switches in the WNB and an even higher number in the SNB networks. Despite its scarce popularity, the
RNB solution is nevertheless interesting, as it can remain the sole possibility to achieve large switch-dimensions in those cases in which technology sets hard bounds to scalability.

We shall point out that this study has on purpose overlooked the wavelength switching domain. Since our investigation is dedicated to space-switching optical fabrics, we make no special assumption on the colors of the optical signals crossing the switches. They can be either all at the same wavelength or they can be WDM connections composed by several multiplexed channels. The general basic condition valid for all the cases we are going to analyze is that the switching operation is always wavelength insensitive, i.e. routing never depends on wavelength. Moreover, multiplexed input signals remain so at the output of the switch and no wavelength conversion operation is carried out. Under this hypotheses, the blocking classification reported above always refers to the sole space-switching and space-multiplexing domain.

\subsection{Physical structure}

A switching architecture is usually composed of a pattern of several optically-interconnected basic Switching Elements (SEs) (as directional couplers), arranged according to a specific topology. The switching fabric can be realized entirely on a unique substrate (single-substrate implementation) or by distributing the SEs among many modules (multiple-substrate implementation).

Advantages of the single-substrate implementation are network compactness and construction simplification. On the other hand, separation on many substrate often allows essential physical-performance improvements. For example, for guided-wave systems the multiple-substrate implementation allows a great reduction in the number of waveguide crossovers (see later), resulting in lower insertion loss and crosstalk. An important drawback of the multiplesubstrate structure is the need for external systems interconnecting the different modules. They are very often implemented by arrays of optical fibers (usually organized in ribbons). If propagation loss on short fiber-spans (as expected to be inside an $\mathrm{OOO}$ ) is normally negligible, coupling loss can be an issue in guided systems due to optical-mode mismatching between fiber and waveguide.

It should be noted that, while any multistage architecture can in principle be implemented on multiple substrate, there are some architectures which are topologically more suitable of being decomposed than others. The multi-substrate implementation is thus more frequent for modular topologies. Incidentally, modular fabrics are often also among the cheapest possible (in terms of number of SEs required to achieve a given switch size). 


\subsection{Signal-transfer impairments}

The signal-transfer performance class includes some parameters related to the degradation suffered by optical signals that cross the switching network.

The first impairment on signal usually considered is attenuation. Contributions to the global attenuation come from several sources: the SEs, the waveguide-bends, the substrate/fiber interfaces, etc., that a given signal crosses inside a switching fabric, all dissipate fractions of its optical power. Thus, attenuation depends on the path the signal has been routed on through the switch: different routings may result in different attenuation values. We define insertion loss $L$ the worst-case value among all those attenuations a signal can possibly face by following a path through the switch. Insertion-loss evaluation is performed from the input to the output port connected by the worst-case path.

Switch loss-features are also characterized by another important parameter: the insertion loss difference $\Delta$. This is defined as the differential attenuation between the most and the least lossy paths through the fabric. Frequently, insertion loss difference is a more critical impairment than insertion loss itself. This is because a high common attenuation can be compensated for with the addition of optical amplifiers, while compensation of insertion loss differences requires a more complex distributed equalization system. A high differential attenuation adversely affects also the optical receiver, since it must be designed for a wider amplitude-dynamic and it must be adapted to react to high fluctuations of the signal-to-noise ratio.

Loss possible contributions are so many that accurate attenuation values can be obtained only by experimental measurements. Since the purpose of our paper is to provide analytical expression for many different switch architectures, we have necessarily to simplify the physical problems resorting to approximated analysis. In the following of the paper we will estimate insertion loss and insertion loss difference by considering only a limited number of phenomena as contributions to attenuation. We are going to complete this discussion in the next section providing specific expressions for $L$ and $\Delta$ for each architecture.

Optical signals are degraded through a space switch also for the accumulation of noise, which lower the Signal-toNoise Ratio (SNR), increasing error probability. Guidedwave switching architectures are seriously affected by this signal impairment, as the majority of integrated-optics systems. Being a switching fabric a multiport device, where many signals converges together in a limited physical space, the dominant noise source is the interference of a given optical channel with the other channels that are simultaneously present in the switch. The crosstalk measures the total optical power transferred to an output of a space-switching matrix from all the inputs different from the one from which comes the expected signal. In order to measure crosstalk, a common approach is to consider the worst case in which all connections are active at the same time. Moreover, crosstalk originating in the interstage interconnections due to optical beam intersection is usually negligible [26]: only power leakage in non-ideal SEs is thus considered. When two connections are active at the same time in the two channels switched by a $2 \times 2 \mathrm{SE}$, each of them receives a so called first-order crosstalk contribution. If a connection crosses alone a $2 \times 2 \mathrm{SE}$, it does not receive first-order crosstalk. However, the free input of the SE may be connected to a free output of another $2 \times 2 \mathrm{SE}$ which is crossed by another active connection. In this case the first connection receives a second-order crosstalk contribution. In our analysis we will always consider crosstalk only up to the second order: this is generally considered sufficiently accurate.

Each SE of the fabric (generally, a directional coupler) is characterized by the so called extinction ratio $m$, which is the ratio of power that is leaked from each channel to the other channel. In the SNR calculations we will use $m$ and the equivalent parameter $X$, which is the inverse of the extinction ratio in $\mathrm{dB}$

$$
X=10 \log _{10} \frac{1}{m}
$$

The majority of papers dealing with optical guided-wave switching fabrics assumes an extinction ratio $m=0.01$ $(X=20 \mathrm{~dB})$. SNR is then evaluated assuming crosstalk as the only source of noise, which is reasonable if we are interested to characterize the fabric itself, considering any other possible noise source (e.g. ASE of optical amplifiers which may be present at inputs or outputs of the fabric) as external. Moreover, ideal optical signals with equal power are assumed to be applied to all the inputs. SNR, as attenuation, generally depends on the path followed by a signal inside the switch. The worst-case SNR among all the possible connections of the switching-network is regarded as the interesting figure, while differential SNR is usually not relevant.

To easily evaluate the entity of SNR a common approximated procedure is followed: second-order crosstalk is only calculated when it is known that no first-order contribution reaches the switch output of the worst-case connection. Otherwise, second-order crosstalk evaluation is useless, since its contribution would be negligible compared to the first-order components. The choice between first and second order calculation is readily taken by inspecting the architecture under exam: first-order crosstalk is evaluated when at least one SE along the worst-case path accommodates two active connections at the same time; second-order crosstalk is computed instead when all the SEs along the worst-case path (and thus in the whole network) are crossed by no more than one connection. The second situation occurs in the so called "dilated" architectures. Under the 
above approximations, in order to evaluate the total first(second-) order crosstalk noise at the output of the worstcase connection, it is sufficient to count the number of SEs along its path that give first- (second-) order crosstalk contributions. If $P_{i}$ is the common input power for all the connections, each of these contributions will be equal to $P_{i} / m\left(P_{i} / m^{2}\right)$. It should be noted that in the approximated conditions the attenuation experienced by a connection will equally apply to both signal and crosstalk-noise contributions and will cancel out of the final SNR. Thus, if $A$ is the number of crosstalk-active (contributing) SEs along the worst-case path, SNR is given by

$$
\mathrm{SNR}=\frac{P_{o}}{P_{\mathrm{xtalk}}}=\frac{1}{A \cdot m^{\gamma}}
$$

where $\gamma=1$ or $\gamma=2$ if the evaluated crosstalk is of the first or the second order, respectively. Usually, SNR is expressed in $\mathrm{dB}$ as follows

$$
\operatorname{SNR}[\mathrm{dB}]=10 \log _{10}\left[\left(\frac{1}{m}\right)^{\gamma} A^{-1}\right]=\gamma X-10 \log _{10} A
$$

In our crosstalk approximated evaluation we have considered only SEs. Actually, in guided-wave architectures two signals also interacts at waveguide crossovers, where a small crosstalk noise is generated. Crossover crosstalk is often difficult to measure and strictly depends not only on waveguide and material properties, but also on the geometry of the intersections (and in particular on the intersection angle). It has been traditionally not considered, being regarded as negligible compared to the SE crosstalk. It can become however a major cause of impairment in those architectures in which the number of crossovers is extremely high. We are currently studying this problem in order to define a less approximate model which takes also crossovers into account in crosstalk evaluations.

We shall finally point out that for the reasons mentioned at the end of Sec. 2.1, in the framework of this paper it is impossible to distinguish between crosstalk at the same (homodyne) or at a different wavelength (heterodyne) of the signal. It is however well known that homodyne and heterodyne crosstalks have very different impacts on the quality of signal.

\subsection{Cost}

The cost of an optical space-switching matrix can be evaluated by counting its elementary components and attributing to each one of them a unitary cost. This evaluation is not always accurate. Especially with integrated optics, costs of the fabrication process (and its production yield) is related to parameters such as substrate area and waveguide width, which are not necessarily directly proportional to the number of SEs or their density. However our rough cost evaluation may be useful to have an idea of the scalability of each particular architecture.

Characteristics correlated to the cost of the architectures comprise: number of $2 \times 2$ switching elements, total number of driver devices.

As already mentioned above, the switching architectures we are considering are obtained by replicating many times a single elementary SE (usually a directional coupler). The number of SEs $S$ necessary to achieve a given switch size is the first total-cost parameter we will consider to compare the switching architectures.

The state of each SE (cross or bar) is electrically controlled by some kind of driving actuator, according to the specific switching technology adopted (electromechanical, thermal, electromagnetic, electrostatic, etc.). Any driver is per-se a source of cost. The number of drivers is not always equal to the number of controlled SEs. In fact, there is a number of architectures (e.g. the tree-type networks) in which many SEs can be controlled by the same driver, since the switching network is devised in such a way that there are groups of SEs which always change their state coherently and simultaneously. Therefore, we have considered the number of drivers $D$ as the second relevant costparameter (of course, $D \leq S$ in any architecture).

\section{Guided-wave switching architectures}

Guided-wave space switches are often fabricated on lithium niobate substrates on which light-guiding structures are created by titanium indiffusion $\left(\mathrm{Ti}: \mathrm{LiNbO}_{3}\right.$ technology)[23]. Various types of electrically-controlled optical SEs can be integrated on the substrate, including electrooptical and thermooptical switches[8]. Complex and very powerful architectures can be realized exploiting $1 \times 2$, $2 \times 1$ and $2 \times 2$ SEs. These devices are relatively reliable and capable of changing their state extremely rapidly, guaranteeing low switching time values. Unfortunately, they suffer high insertion loss and possible polarization dependence.

In order to evaluate attenuation, three types of contributions have been taken into account: power dissipation of each SE, waveguide-to-fiber and fiber-to-waveguide coupling $\operatorname{loss}^{2}$ and crossover loss. The last contribution is a peculiarity of waveguided architectures: in these fabrics, SEs and interconnection-stage waveguides are integrated on a common substrate. Unlike integrated electronic circuits, in which connections between the various components can be made at multiple levels and separated by dielectric material, in integrated optics waveguides physically cross each other on the same substrate. Waveguide intersection is the cause of many undesirable effects, one of which is power loss.

\footnotetext{
${ }^{2}$ It should be noted that in all the architectures considered, coupling loss is path-independent (equal for all the paths).
} 
Let us consider the worst-case path, that crosses $K$ SEs, $I$ (fiber-waveguide + waveguide-fiber) interfaces and $W$ waveguide crossovers. The total insertion loss (in $\mathrm{dB}$ ) is given by

$$
L[\mathrm{~dB}]=s \cdot K+c \cdot I+w \cdot W
$$

where $s$ is the loss of a SE, $c$ is the loss of a fiber-waveguide (waveguide-fiber) interface and $w$ is the loss experienced by a signal propagating along a waveguide each time this crosses another waveguide. The insertion loss difference is calculated by subtracting from $L$ the loss (in $\mathrm{dB}$ ) obtained applying the above equation to the best-case path.

\subsection{Switching architectures}

We have considered 22 different guided-wave network architectures and we have reported their characteristics into three different tables, grouping them according to their blocking features. Fig. 1, 2 and 3 concern SNB, WNB and RNB architectures, respectively. For each architecture, we have analyzed the seven features introduced in Sec. 2: physical structure, insertion loss, insertion loss difference, SNR, number of SEs, number of drivers and number of waveguide crossovers. The choice of the physical structure type has been made according to the intrinsic properties of each architecture. For modular networks we have preferred multisubstrate realizations while we have chosen single-substrate structures where it has been difficult or useless to identify simple modules inside the switch. In the tables, letters $\mathrm{S}$ and $\mathrm{M}$ indicate single-substrate and multiple-substrate structures, respectively. When the multiple-substrate configuration has been chosen for a certain architecture, the last column of the three tables indicates the additional number of waveguide crossovers between the input and output ports that the worst-case connection would cross if the same architecture had been realized on a single substrate.

In the tables, values of insertion loss, insertion loss difference and SNR are expressed in $\mathrm{dB}$ while the other values are dimensionless numbers. All the parameters are given as functions of the number of inputs and outputs of the architectures (the size of the network) $N$.

Let us list the architectures we have considered, providing for each the reference to the paper in which it has been presented:

- SNB: classical Clos [4]; Double Layer [16]; Dilated Double Layer [16]; Extended Baseline [29]; Strictsense non-blocking NWN (named here NWN-S) [27]; the numerous class of tree-type networks comprising a number of architectures, as indicated in Fig. 1 $[22,17,11,10,9]$.

- WNB: classical Crossbar [7]; Double Crossbar [14]; Modified Double Crossbar [12]; Wide-sense nonblocking NWN (named here NWN-W) [26].
- RNB: classical Benes [1]; Dilated Benes [18]; Modified Dilated Benes [13]; classical Slepian-Duguid [20]; N-Stage Planar [24].

The association of the Crossbar architecture to the widesense non-blocking network class needs an additional comment. The optical guided-wave implementation of the Crossbar network is usually realized by using $N^{2} 2 \times 2$ SEs, where every SE is equivalent to a crosspoint of the electronic network. Since every SE has two possible active inlets and two possible switching states, such construction offers multiple routes for various i/o pairs. Only one of these possible routes guarantees the non-blocking condition for the network, being the others possible causes of blocking states. So, a routing algorithm must exist (although very simple) that guarantees network non-blockingness. In this way, the network is wide-sense non-blocking. However, the border between SNB and WNB networks is so thin that the same architecture becomes SNB considering a different type of SEs. For example, 2-D MEMS Crossbar systems with single-face-reflective micromirrors fall in the SNB category. In this case, in fact, there is a unique possible path between every input and output, making blocking states impossible without the need of any routing algorithm.

In Clos and Slepian-Duguid networks, given a value of $N$, another independent parameter, $n$, must be chosen in order to fully specify the topology. $n$ is the number of input ports of the switching matrices of the first stage (the number of output ports of the third stage matrices is chosen equal to $n$ ). We have chosen $n=\sqrt{N / 2}$, a value which minimizes the total number of SEs.

For the multiple-substrate implementations of the Clos and Slepian-Duguid architectures, we have assumed that guided-wave Crossbar networks are used to realize each module of the three stages: modules are connected by fibers arranged in EGS patterns.

\subsection{Architecture comparison}

We are now going to numerically compare the various architectures on the basis of the described characteristics. The values of the physical parameters $s, c, w$ and $X$ have been chosen according to the following considerations. $s$ depends on the type of SEs employed. A typical value for directional couplers is $s=0.5 \mathrm{~dB}$. It is convenient, however, to consider the increased value $s=1 \mathrm{~dB}$ to keep waveguide-propagation loss into account. This choice is based on the assumption that the path length of a signal across the switching architecture is roughly proportional to the number of crossed SEs. Realistically, $c$ could be around $1.5 \mathrm{~dB}$, but we have chosen the more conservative value $c=2 \mathrm{~dB}$. Extinction ratio is strongly dependent on the switching element type. In literature, a value of $X=20$ $\mathrm{dB}$ is suggested for directional-coupler-based switches [16]. 


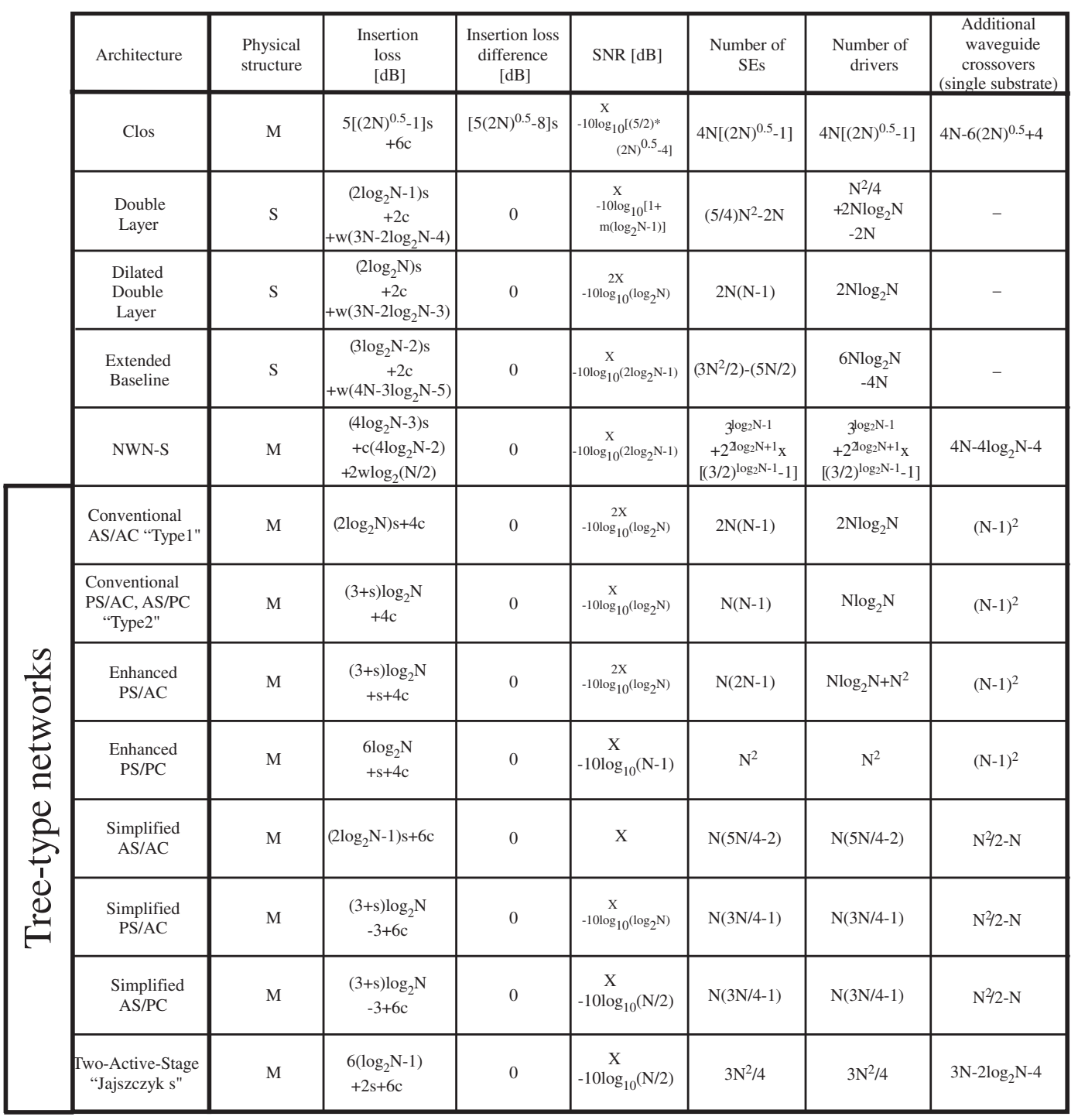

Figure 1. Complete characteristics of strict-sense non-blocking guided-wave architectures.

\begin{tabular}{|c|c|c|c|c|c|c|c|}
\hline Architecture & $\begin{array}{l}\text { Physical } \\
\text { structure }\end{array}$ & $\begin{array}{c}\text { Insertion } \\
\text { loss } \\
{[\mathrm{dB}]}\end{array}$ & $\begin{array}{c}\text { Insertion loss } \\
\text { difference } \\
{[\mathrm{dB}]}\end{array}$ & SNR [dB] & $\begin{array}{c}\text { Number of } \\
\text { SEs }\end{array}$ & $\begin{array}{c}\text { Number of } \\
\text { drivers }\end{array}$ & $\begin{array}{c}\text { Additional } \\
\text { waveguide } \\
\text { crossovers } \\
\text { (single substrate) }\end{array}$ \\
\hline Crossbar & $S$ & $(2 \mathrm{~N}-1) \mathrm{s}+2 \mathrm{c}$ & $(2 \mathrm{~N}-2) \mathrm{s}$ & $\frac{\mathrm{X}}{-10 \log _{10}(\mathrm{~N}-1)}$ & $\mathrm{N}^{2}$ & $\mathrm{~N}^{2}$ & - \\
\hline $\begin{array}{c}\text { Double } \\
\text { Crossbar }\end{array}$ & $S$ & $\begin{array}{l}(\mathrm{N}+1) \mathrm{s}+2 \mathrm{c} \\
+\mathrm{wN}(\mathrm{N}-1)\end{array}$ & 0 & $\begin{array}{c}2 \mathrm{X} \\
-10 \log _{10}(\mathrm{~N}-1)\end{array}$ & $2 \mathrm{~N}^{2}$ & $2 \mathrm{~N}^{2}$ & - \\
\hline $\begin{array}{l}\text { Modified } \\
\text { Double } \\
\text { Crossbar }\end{array}$ & $\mathrm{S}$ & $\begin{array}{c}(3 \mathrm{~N} / 4+2) \mathrm{s}+2 \mathrm{c} \\
+\mathrm{w}\left(\mathrm{N} \log _{2} \mathrm{~N}+\mathrm{N} / 4-2\right)\end{array}$ & 0 & $\stackrel{2 \mathrm{X}}{-\operatorname{lol}_{10}\left(\log _{2} \mathrm{~N}-1\right)}$ & $2 \mathrm{~N}^{2}$ & $3 \mathrm{~N}^{2} / 2$ & - \\
\hline NWN-W & M & $\begin{array}{l}\left(4 \log _{2} \mathrm{~N}-3\right) \mathrm{s} \\
+\mathrm{c}\left(2+4 \log _{2} \mathrm{~N} / 2\right)\end{array}$ & $\left(2 \log _{2} \mathrm{~N}-2\right) \mathrm{s}$ & $\underset{-10 \log _{10}\left(2 \log _{2} \mathrm{~N}-1\right)}{\mathrm{X}}$ & $\begin{array}{c}13\left(3^{\log _{2} \mathrm{~N}-1}\right) \\
-6 \mathrm{~N}\end{array}$ & $\begin{array}{l}4 \mathrm{~N}(3 / 2)^{\log _{2} \mathrm{~N}-1} \\
-4 \mathrm{~N}+3^{\log _{2} \mathrm{~N}-1}\end{array}$ & $4 \mathrm{~N}-4 \log _{2} \mathrm{~N}-4$ \\
\hline
\end{tabular}

Figure 2. Complete characteristics of wide-sense non-blocking guided-wave architectures. 


\begin{tabular}{|c|c|c|c|c|c|c|c|}
\hline Architecture & $\begin{array}{l}\text { Physical } \\
\text { structure }\end{array}$ & $\begin{array}{c}\text { Insertion } \\
\text { loss } \\
{[\mathrm{dB}]}\end{array}$ & $\begin{array}{c}\text { Insertion loss } \\
\text { difference } \\
{[\mathrm{dB}]}\end{array}$ & SNR [dB] & $\begin{array}{c}\text { Number of } \\
\text { SEs }\end{array}$ & $\begin{array}{c}\text { Number of } \\
\text { drivers }\end{array}$ & $\begin{array}{c}\text { Additional } \\
\text { waveguide } \\
\text { crossovers } \\
\text { (single substrate) }\end{array}$ \\
\hline Benes & S & $\begin{array}{c}\left(2 \log _{2} \mathrm{~N}-1\right) \mathrm{s} \\
+2 \mathrm{c} \\
+\mathrm{w}\left(2 \mathrm{~N}-2 \log _{2} \mathrm{~N}-2\right) \\
\end{array}$ & 0 & $\underset{-10 \log _{10}\left(2 \log _{2} \mathrm{~N}-1\right)}{\mathrm{X}}$ & $(\mathrm{N} / 2)\left(2 \log _{2} \mathrm{~N}-1\right)$ & $(\mathrm{N} / 2)\left(2 \log _{2} \mathrm{~N}-1\right)$ & - \\
\hline $\begin{array}{l}\text { Dilated } \\
\text { Benes }\end{array}$ & S & $\begin{array}{c}\left(2 \log _{2} \mathrm{~N}\right) \mathrm{s} \\
+2 \mathrm{c} \\
+\mathrm{w}\left(4 \mathrm{~N}-2 \log _{2} \mathrm{~N}-5\right)\end{array}$ & 0 & $\begin{array}{c}2 \mathrm{X} \\
-10 \log _{10}\left[\left(2 \log _{2} \mathrm{~N}-1\right)\right. \\
\left.\log _{2} \mathrm{~N}\right]\end{array}$ & $2 \mathrm{~N} \log _{2} \mathrm{~N}$ & $2 \mathrm{~N} \log _{2} \mathrm{~N}$ & - \\
\hline $\begin{array}{l}\text { Modified } \\
\text { Dilated } \\
\text { Benes } \\
\end{array}$ & S & $\begin{array}{c}\left(2 \log _{2} N+1\right) \mathrm{s} \\
+2 \mathrm{c} \\
+\mathrm{w}\left(4 \mathrm{~N}-2 \log _{2} \mathrm{~N}-5\right) \\
\end{array}$ & 0 & $\begin{array}{c}2 \mathrm{X} \\
-10 \log _{10}\left[\left(\log _{2} \mathrm{~N}-1\right)\right. \\
\left.\log _{2} \mathrm{~N}\right]\end{array}$ & $2 \mathrm{~N}\left(\log _{2} \mathrm{~N}+1\right)$ & $2 \mathrm{~N}\left(\log _{2} \mathrm{~N}+1\right)$ & - \\
\hline $\begin{array}{l}\text { Slepian- } \\
\text { Duguid }\end{array}$ & M & $\begin{array}{c}{\left[4(2 \mathrm{~N})^{0.5}-3\right] \mathrm{s}} \\
+6 \mathrm{c}\end{array}$ & $2\left[2(2 \mathrm{~N})^{0.5}-3\right] \mathrm{s}$ & $\begin{array}{c}\mathrm{X} \\
-10 \log _{10}^{\left[2^{* *}\right.} \\
\left.(2 \mathrm{~N})^{0.5}-3\right]\end{array}$ & $(2 \mathrm{~N})^{1.5}$ & $(2 \mathrm{~N})^{1.5}$ & $2 \mathrm{~N}-3(2 \mathrm{~N})^{0.5}+2$ \\
\hline $\begin{array}{l}\text { N-Stage } \\
\text { Planar }\end{array}$ & S & $\mathrm{Ns}+2 \mathrm{c}$ & $(\mathrm{N} / 2) \mathrm{s}$ & $\begin{array}{c}\mathrm{X} \\
-10 \log _{10} \mathrm{~N}\end{array}$ & $\mathrm{~N}(\mathrm{~N}-1) / 2$ & $\mathrm{~N}(\mathrm{~N}-1) / 2$ & - \\
\hline
\end{tabular}

Figure 3. Complete characteristics of rearrangeable non-blocking guided-wave architectures.

Despite the fact that modern fabrication techniques achieve a much lower level of crosstalk, we prefer keeping $X=20$ $\mathrm{dB}$ as reference, to be conservative. Finally, considering $2 \mu \mathrm{m}$-wide waveguides that intersect each other at an average angle of $50^{\circ}$, a realistic value for signal loss due to every single crossover is $w=45 \mathrm{mdB}$ [2]. It is important to say that such value refers to a specific case (InP-based photonic integrated circuits) and that different materials could give different results. However, the contribution of a single crossover to the total loss is so low that different values of $w$ would give rise to significative differences only with very large architectures.

In the following, we show graphs for some network characteristics in which many architectures of the three classes (SNB, WNB and RNB) are plotted: this allows an easy observation of the differences among architectures belonging to the various classes. Curves corresponding to SNB architectures have been drawn with a continuous line, while a long-dashed line is used for WNB and a short-dashed line for RNB.

Fig. 4 shows the insertion loss. We can see that, in general, guided-wave architectures have large attenuation values also for small network dimensions. The loss increases very rapidly with the size. Large dimension networks can be used only if coupled with optical amplifiers capable to compensate the loss introduced by the switch. In the graph, some architectures display a linear trend while others present a parabolic trend, the latter being a relevant obstacle to scalability. The network architecture with the smallest value of insertion loss is the Benes network for $N \leq 64$, while for $N>64$ the conventional AS/AC becomes the best one. The explanation of this behavior is that every signal in a Benes network must pass through $2 \log _{2} N-1$ SEs while the same signal must pass through
$2 \log _{2} N$ SEs in a conventional AS/AC architecture. Since we have chosen the single-substrate Benes implementation, the effect of the crossovers is rather negligible for small network sizes. On the opposite, when the network dimension increases, the signal loss due to the large number of waveguide crossovers cannot be neglected anymore and so the total attenuation becomes high. In the conventional AS/AC waveguide crossover is not relevant, because of the presence of optical fibers between diverse substrates. Fig. 4 does not show relevant differences in the insertion loss values for the architectures belonging to the three non-blocking classes. In other words, we can find architectures with a relative low or high insertion loss independently of the blocking property of the network (i.e. if it is SNB, WNB or RNB). It is interesting to note that several switching networks are roughly equivalent for small values of $N$ under the loss point of view. For example, Benes, Double Layer, Dilated Double Layer, Dilated Benes and Modified Dilated Benes have similar attenuation values for $N \leq 32$. On the opposite, only the Conventional AS/AC architecture is capable to keep insertion loss on acceptable values when $N$ becomes high. All the other architectures, in fact, reach very high attenuation levels because of the large number of SEs crossed by the connections and mainly because of the huge number of waveguide crossovers along the paths.

Only five architectures present a differential attenuation among input-output connections. For these architectures the differential attenuation rapidly increases to very large amounts, causing high insertion loss differences. The Crossbar architecture has the worst behavior under this aspect because the insertion loss difference is almost equal to the insertion loss value. This is due to the fact that the shortest path inside the matrix always crosses just one SE and hence it is independent of the network dimension. In a 


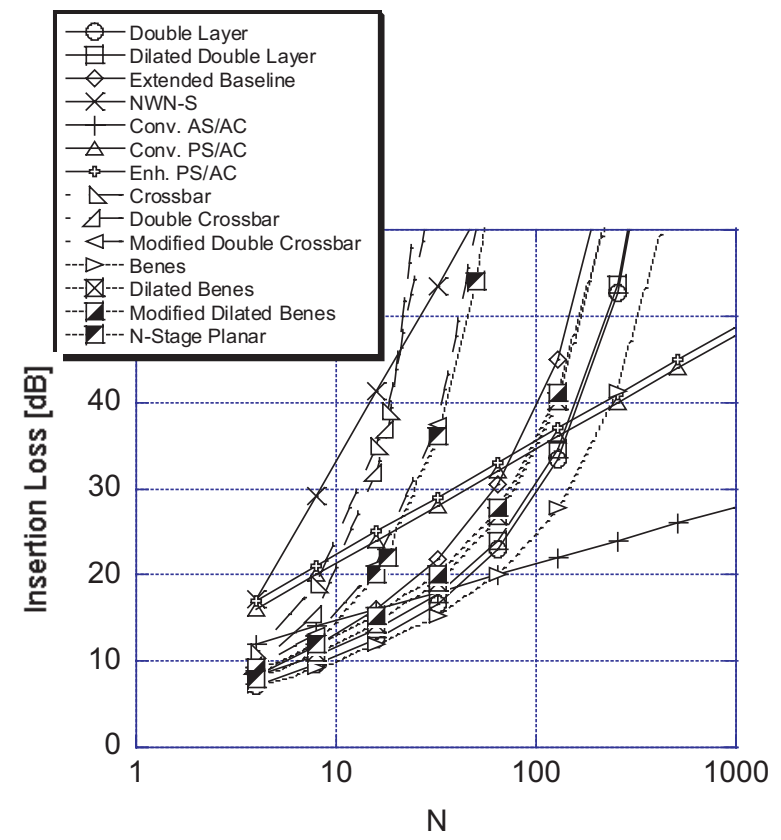

Figure 4. Insertion loss for various guidedwave switching architectures.

large dimension Crossbar, the longest path suffers losses of the order of many tens of $\mathrm{dB}$ while the attenuation on the shortest path, crossing only one SE, becomes almost negligible. This large insertion loss difference would impose so much stress on the optical receiver amplitude-dynamic that the use of large Crossbar architectures for switching becomes practically unfeasible. Clos and Slepian-Duguid architectures decrease the insertion loss difference with respect to the Crossbar network, but improvements are limited by the use of Crossbar networks in every block they are composed of. N-Stage Planar networks have a particular behavior: it can happen that $N-2$ i/o connections (out of $N$ ) cross exactly $N$ SEs, while the other two connections cross $N / 2$ SEs. For this reason in this type of networks the insertion loss difference is strongly dependent on the network size $N$ and increases linearly with it. NWN-W networks have acceptable values of differential loss, especially for small architectures. Fortunately, all the other described switching networks have an insertion loss difference exactly equal to zero.

In Fig. 5 SNR is plotted as a function of the network size. Best results are obtained by the architectures which guarantee zero first-order crosstalk. These are: Modified Double Crossbar (having the best behavior in absolute), Enhanced PS/AC, Modified Dilated Benes, Dilated Benes and Double Crossbar. The case of the Double Layer Networks is very interesting: they have an almost constant high value of SNR, despite they belong to the class of non-zero firstorder crosstalk networks. As the previously analyzed characteristics, also the signal-to-noise ratio is independent of the connection property of the network. For example, the three architectures that present the best SNR performance are wide-sense, strict-sense and rearrangeable non-blocking respectively. It could be proved that the minimum acceptable SNR to achieve a bit error rate of $10^{-9}$ is about 11 $\mathrm{dB}$ [10]. For this reason, all the architectures with a lower SNR value are hardly of practical use. Unfortunately, many guided-wave switching architectures have SNR below 11 $\mathrm{dB}$ also with a very small network size. Some architectures have SNR also below $0 \mathrm{~dB}$, which means that the noise power is larger than the signal power in the optical signals outgoing by the switch. So, if the goal is to construct a large architecture, we have a very limited number of wave-guided switching architectures to rely upon.

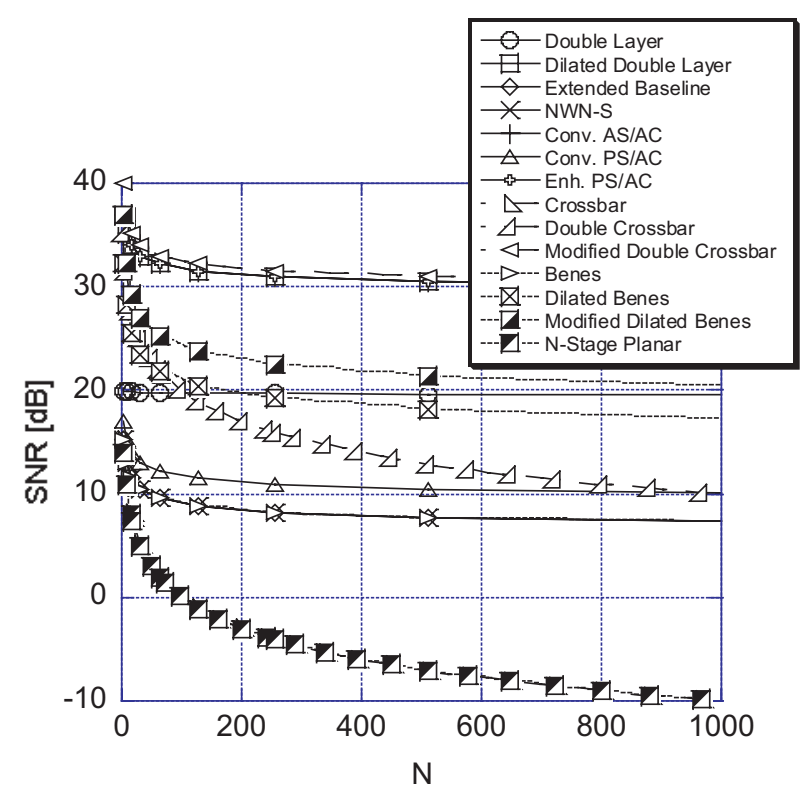

Figure 5. Signal-to-Noise characteristics for various guided-wave switching architectures.

Let us now consider the cost parameters. The analyzed architectures behave very differently under the point of view of the total number of SEs (Fig. 6). As it is well known, the very large number of SEs, increasing with the square of the network size, is one of the main problems of the Crossbar. Fig. 6 shows that many architectures require a number of SEs even greater than that of the Crossbar. On the other hand, there are some architectures whose complexity increases slowly with $N$, resulting nicely scalable. Two important aspects can be noted in the graph. First, an enormous difference in the number of SEs exists between the 
category of RNB architectures and the other two categories. Second, the difference in the number of SEs between SNB and WNB networks is not very relevant. So, if the disruption of optical connections inside the space switch is not a problem, RNB networks are strongly to be preferred. On the opposite, if connection rearrangement has to be avoided, SNB architectures are the best choice since their control is very easy and their cost is similar to that of WNB architectures.

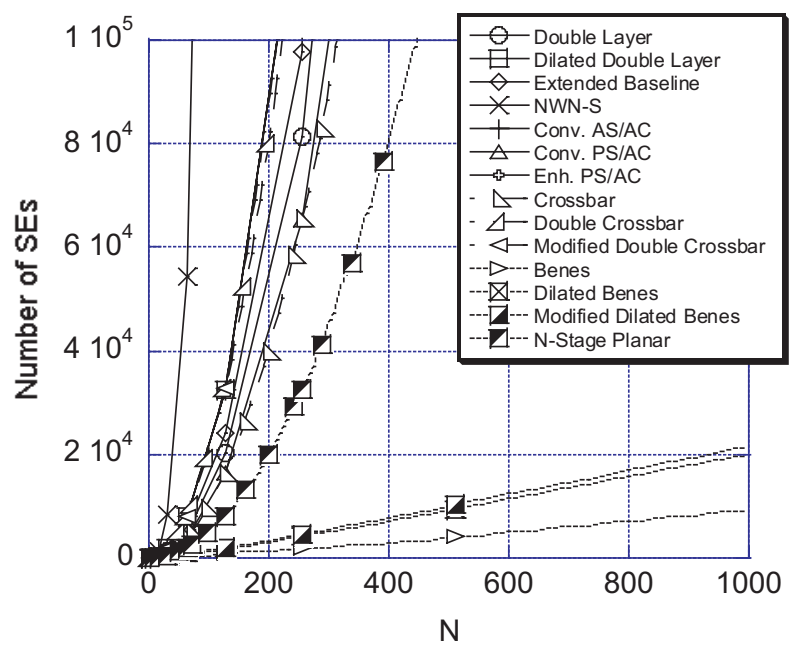

\section{Figure 6. Number of SEs required for various guided-wave switching architectures.}

Let us now consider the number of drivers with the size of the networks. Dilated Double Layer, Extended Baseline, Conventional AS/AC and PS/AC architectures, interestingly, require a very small number of drivers even with a large number of SEs. This is because in tree-type networks all the SEs belonging to the same column in a splitter or in a combiner change their state at the same time and then they can be driven by a common control signal. In the other two types of networks, even if there are neither splitters nor combiners, some SEs change their state at the same time and can be driven by the same system. RNB architectures ensure the best performance also for this characteristic. The sole exception is represented by the N-Stage Planar networks that require a large number of SEs and drivers, even greater than that of many SNB architectures.

Optical signals passing through single-substrate architectures usually encounter a very high number of waveguide crossovers. Under this aspect, a multiple-substrate implementation is the best solution. Fig. 7 represents the maximum number of waveguide crossovers between input and output ports in various networks with different values of $N$. We have considered only architectures for which the phys- ical structure reported in Fig. 1-3 is the single-substrate type, except the NWN-S. For networks having a number of crossovers in the order of some thousands, the attenuation term due to waveguide crossovers cannot be neglected in the expression of the insertion loss.

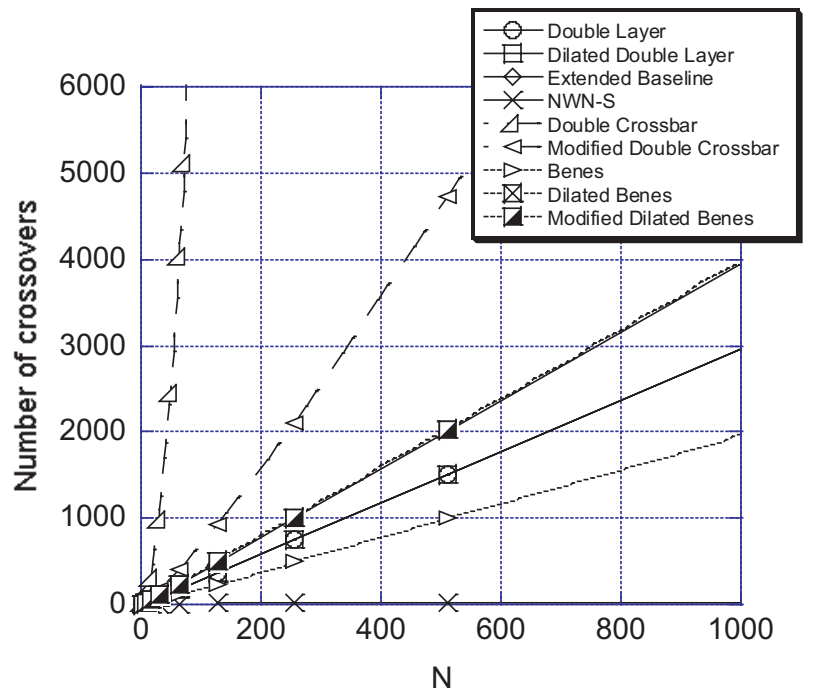

Figure 7. Number of waveguide crossover for various guided-wave switching architectures.

\section{Conclusion}

Optical switching node design is a complex task, perhaps even more complex than the design of electronic switches. In the electronic domain, the unique target is to minimize the total number of switching elements required by the network. On the opposite, in the optical domain, there are other additional parameters to be optimized as system attenuation, number of crossovers, and signal-to-noise ratio. Therefore, the optimal architectures for photonic switching are not necessarily the optimal architectures for electronic switching, since the two applications undergo different sets of constraints. This study addresses the considerations involved in selecting a guided-wave architecture to be used as an optical space switch fabric. The relevant characteristics of optical switching architectures have been defined and a great number of previously proposed structures have been reviewed. To successfully compare different architectures, many issues have still to be investigated. However, we can say in general that there is no best switching architecture in absolute, but only architectures most suitable for each specific application, depending on the relative constraints the specific application requirements impose to the various characteristics. If all-optical switching will actually 
prove to be cost effective compared to electronic switching in the future, some of the switching architectures reviewed in this paper could be probably adopted in the next generation OXCs.

\section{References}

[1] V. E. Benes. Mathematical Theory of Connecting Networks and Telephone Traffic. New York: Academic Press, 1965.

[2] H. G. Bukkems, C. G. P. Herben, M. K. Smit, F. H. Groen, and I. Moerman. Minimization of the Loss of Intersecting Waveguides in InP-Based Photonic Integrated Circuits. IEEE Photonics Technology Letters, 11(11):14201422, November 1999.

[3] D. K. Cheng, Y. Liu, and G. J. Sonek. Optical switch based on thermally-activated dye-doped biomolecular thin films. IEEE Photon. Technol. Lett., 7:366-369, April 1995.

[4] C. Clos. A study of non-blocking switching networks. Bell System Tech. Journal, 32:406-424, Mar. 1953.

[5] A. Dugan, L. Lightworks, and J. C. Chiao. The optical switching spectrum: A primer on wavelength switching technologies. Telecommun. Magazine, May 2001.

[6] S. Hardy. Liquid-crystal technology vies for switching applications. Lightwave, pages 44-46, December 1999.

[7] H. S. Hinton. A nonblocking optical interconnection network using directional couplers. In GLOBECOM 1984, volume 26, pages 26.5.1-26.5.5, 1984.

[8] H. S. Hinton. Photonic switching using directional couplers. IEEE Communication Magazine, 25(5):16-26, May 1987.

[9] A. Jajszczyk. A Class of Directional-Coupler-Based Photonic Switching Networks. IEEE Transactions on Communications, 41:599-603, April 1993.

[10] A. Jajszczyk and H. T. Mouftah. Tree architectures for photonic switching. In Global Telecommunications Conference, volume 3, pages 1605-1609, December 1992.

[11] A. Jajszczyk and H. T. Mouftah. Tree-Type Photonic Switching Networks. Network, 9:10-16, January 1995.

[12] W. Kabacinski. Modified Double Crossbar Photonic Switching Networks. In Electrotechnical Conference MELECON 98, 9th Mediterranean, volume 2, pages 754-758, 1998.

[13] W. Kabacinski. Modified Dilated Benes Networks for Photonic Switching. IEEE Transactions on Communications, 47(8):1253-1259, August 1999.

[14] M. Kondo, N. Takado, K. Komatsu, and Y. Ohta. 32 switch elements integrated low-crosstalk $\mathrm{LiNbO}_{3} 4 \times 4$ optical matrix switch. In IOOC-ECOC '85, pages 361-364, Venice, 1985.

[15] L.-Y. Lin, E. L. Goldstein, and R. W. Tkach. Free-Space Micromachined Optical Switches for Optical Networking. Journal of Selected Topics in Quantum Electronics, 5(1):49, January-February 1999.

[16] C.-C. Lu and R. A. Thompson. The Double-Layer Network Architecture for Photonic Switching. Journal of Lightwave Technology, 12(8):1482-1489, August 1994.

[17] H. Okayama, A. Matoba, R. Shibuya, and T. Ishida. Optical Switch Matrix With Simflified $\mathrm{N} \times \mathrm{N}$ Tree Structure. Journal of Lightwave Technology, 7(7):1023-1028, July 1989.
[18] K. Padmanabhan and A. Netravali. Dilated Networks for Photonic Switching. IEEE Transactions on Communications, 35:1357, December 1987.

[19] G. I. Papadimitriou, C. Papazoglou, and A. S. Pomportsis. Optical Switching: Switch Fabrics, Techniques, and Architectures. Journal of Lightwave Technology, 21(2):384-404, February 2003.

[20] A. Pattavina. Switching Theory: Architectures and Performance in Broadband ATM Networks. John Wiley \& Sons, Baffin Lane, Chichester, West Sussex, England, first edition, 1998.

[21] D. A. Smith, A. Alessandro, J. E. Baran, D. J. Fritz, J. L. Jackel, and R. S. Chakravarthy. Multiwavelength performance of an adopized acoust-optic switch. Journal of Lightwave Technology, 14:2044-2051, September 1996.

[22] R. A. Spanke. Architecture for Large Nonblocking Optical Space Switches. IEEE Journal of Quantum Electronics, 22(6):964-967, June 1986.

[23] R. A. Spanke. Architecture for Guided-Wave Optical Space Switching Systems. IEEE Communication Magazine, 25(5):42-48, May 1987.

[24] R. A. Spanke and V. Benes. An N-stage planar optical permutation network. Applied Optics, 26, April 1987.

[25] T. E. Stern and K. Bala. Multiwavelength Optical Networks: A Layered Approach. Addison-Wesley, 1999.

[26] F. M. Suliman, A. B. Mohammad, and K. Seman. A New Nonblocking Photonic Switching Network. In GLOBECOM '01, volume 4, pages 2071-2076, 2001.

[27] F. M. Suliman, A. B. Mohammad, and K. Seman. A Space Dilated Lightwave Network - A New Approach. In International Conference on Telecommunications, volume 2, pages 1675-1679, February-March 2003.

[28] A. Ware. New photonic-switching technology for all-optical networks. Lightwave, pages 92-98, March 2000.

[29] C.-S. Wu, G.-K. Ma, and B.-S. P. Lin. Extended Baseline Architecture for Nonblocking Photonic Switching. Journal of Lightwave Technology, 15(5):771-778, May 1997. 